Honam Mathematical J. 33 (2011), No. 3, pp. 341-346

http://dx.doi.org/10.5831/HMJ.2011.33.3.341

\title{
ON THE ENTIRE SERIES WITH FINITE LOGARITHMIC ORDER
}

\author{
Peter Tien-Yu Chern And In-Soo Kim
}

\begin{abstract}
We deal with a kind of impossible decomposition of some entire functions in terms of a certain form.
\end{abstract}

$L \ddot{u}[\mathrm{~L}]$ obtained an impossible decomposition for some composite entire functions, we state it as follows:

Theorem A. ([L, p.95, Theorem 2.1.]) If $h(z)$ is a transcendental entire function and $g$ is an entire function, then the decomposition of the composite function $g(h)(z)$ may not have the following form:

$$
P(z) g(z)+Q(z)
$$

where $P$ and $Q$ are polynomials.

These $P$ and $Q$ are polynomials in the above Theorem A because $L \ddot{u}^{\prime} s$ statement is for any transcendental entire function $h(z)$. It seems that if one can specify the growth of $h(z)$, then we may make the growth of $P$ much close to the growth of $h(z)$.

The purpose of the present paper is to find results like above Theorem A for functions with more specific growths. To this end we need several terminologies.

Definitions. Let $S(r), \varphi(r)$ and $\psi(r)$ be nonnegative unbounded increasing functions defined for $r>0$, and $\varphi^{\prime}(r)$ and $\psi^{\prime}(r)$ exist, and they are positively continuous except on a set of isolated points. We say $S(r)$ is of $\varphi$-order $\lambda$ (see [C2]), if $\lambda=\limsup _{r \rightarrow+\infty} \log S(r) / \log \varphi(r)$; in case

Received June 14, 2011. Accepted July 8, 2011.

2000 Mathematics Subject Classification. Primary 30D30, 30D35.

Key words and phrases. Impossible decomposition, Lower $\psi$ - order, Composition function.

This paper was supported by research funds of Chonbuk National University in 2009 . 
$\varphi(r)=r, \varphi$-order is the usually order for $S(r)$, see [N]; in case $\varphi(r)=$ $\log r, \varphi$-order is the logarithmic order for $S(r)$ (see [C1]). We say that an entire function $f$ is of $\varphi$-order $\lambda$, if $\log M(r, f)$ has $\varphi$-order $\lambda$, where $M(r, f)$ denotes the maximum module function of $f$.

Just before, we said that $S(r)$ is of lower $\psi$-order $\rho$, if $\rho=\liminf _{r \rightarrow+\infty} \log S(r) /$ $\log \psi(r)$, in case $\psi(r)=$ r, the lower $\psi$-order is the usually lower order for $S(r)$ [N]; in case $\psi(r)=\log r$, the lower $\psi$-order is the lower logarithmic order for $S(r)$. We say that an entire function $f$ is of lower $\psi$-order $\rho$, if $\log M(r, f)$ has lower $\psi$-order $\rho$.

Results of this article are stated as follows:

Theorem 1. If $h(z)$ is an entire function with infinite lower logarithmic order and with finite positive lower $\psi-\operatorname{order} \rho$, then for any non-constant entire function $g(z)$ the decomposition of the composite function $g(h)(z)$ may not have the following form

$$
P(z) g(z)+Q(z),
$$

where $\mathrm{P}, \mathrm{Q}$ are entire functions, $P$ has a $\psi$-order less than $\rho$ and $M(r, Q)=\mathrm{o}(M(r, g))$ as $r \longrightarrow+\infty$.

Theorem 2. If $h(z)$ is an entire function and $h(z)$ has a finite positive lower logarithmic order $\rho>1$, then for any non-constant entire function $g(z)$ the decomposition of the composite function $g(h)(z)$ may not have the following form

$$
P(z) g(z)+Q(z),
$$

where $P$ and $Q$ are entire functions and $P$ has a lower logarithmic order less than $\rho$ and $M(r, Q)=\mathrm{o}(M(r, g))$ as $r \longrightarrow+\infty$.

\section{Preliminaries}

Lemma 1. (Bohr's Lemma[B]) Let $f(z)$ be a function analytic in the open unit disk $D$ and satisfy two conditions: $f(0)=0$ and $M(1 / 2, f) \geq$ 1. Then in the disk $D$ the function $f(z)$ assumes every value $w$ on a circle $|w|=r$ with $r>A$, where $A$ is a positive numerical constant. 
Lemma 2. Let $h$ and $g$ be entire functions. If $h$ has a lower $\psi$-order $\rho$, then for small positive number $\varepsilon$ we have the following:

$$
\log M\left(r^{(\psi(r))^{\rho-\varepsilon}}, g\right) \geq \frac{(\psi(r))^{\rho-\varepsilon}}{\log r} \log M(2 r, g) .
$$

\section{Proofs}

The Proof of Lemma 2. Assume that $h$ has a lower $\psi$-order $\rho$. Given small positive number $\varepsilon_{1}$, there exists a positive number $r_{\varepsilon_{1}}$ such that if $r \geq r_{\varepsilon_{1}}$, then

$$
\log M(r, h)>\psi(r)^{\rho-\varepsilon_{1}} .
$$

For each $r$ with $0<r<1$, we define a function $H: D \rightarrow \mathbb{C}$,

$$
H(z)=\frac{h(r z)-h(0)}{M\left(\frac{r}{2}, h-h(0)\right)},
$$

where $\mathbb{C}$ is the complex plane. Then $H(z)$ is analytic in $D$ and satisfies the following: $H(0)=0$ and $M\left(\frac{1}{2}, H\right)=1$.

Applying Bohr's result(Lemma 1), there exists a number $R^{\prime} \geq A>0$, where $A$ is constantly given as in Bohr's Lemma such that $H$ assumes every value $w$ in the circle $|w|=R^{\prime}$. Put $R=R^{\prime} M\left(\frac{r}{2}, h-h(0)\right)$, then we have the following:

$$
R>A(M(r / 2, h)-|h(0)|) .
$$

Since $g$ is an entire function, there exists a point $\mathrm{w}_{0} \in \mathbb{C}$ such that

$$
\operatorname{Max}_{|\mathrm{w}-h(0)|=R}|g(\mathrm{w})|=g\left(\mathrm{w}_{0}\right) .
$$

In $|z|<r$, there exists a point $z_{0}$ such that $h\left(z_{0}\right)=\mathrm{w}_{0}$ and

$$
M(r, g(h)) \geq\left|g(h)\left(z_{0}\right)\right| \geq M(R-|h(0)|, g) .
$$

Further, there is a positive number $A_{1}$ such that

$$
R-|h(0)|>A_{1} M(r / 2, h)>A_{1} e^{(\psi(r / 2))^{\rho-\varepsilon_{1}}} .
$$

Thus

$$
M(r, g(h))>M(R-|h(0)|, g)>M\left(A_{1} e^{\left(\psi\left(\frac{r}{2}\right)\right)^{\rho-\varepsilon_{1}}}, g\right) .
$$


By choosing $\varepsilon_{1}$, we may assume $A_{1}=1$. Taking a logarithm for above inequality, we have

$$
\log M(r, g(h)) \geq \log M\left(e^{\left(\psi\left(\frac{r}{2}\right)\right)^{\rho-\varepsilon_{1}}}, g\right) .
$$

Since $\log M(r, g)$ is convex in $\log r$, for $r_{0}<r<e^{\left(\psi\left(\frac{r}{2}\right)\right)^{\rho-\varepsilon_{1}}}$ we have the following:

(9) $\log M(r, g) \leq \frac{\psi\left(\frac{r}{2}\right)^{\rho-\varepsilon_{1}}-\log r}{\psi\left(\frac{r}{2}\right)^{\rho-\varepsilon_{1}}-\log r_{0}} \log M\left(r_{0}, g\right)+\frac{\log r-\log r_{0}}{\psi\left(\frac{r}{2}\right)^{\rho-\varepsilon_{1}}-\log r_{0}} \log M\left(e^{\left(\psi\left(\frac{r}{2}\right)\right)^{\rho-\varepsilon_{1}}}, g\right)$.

Thus

$$
\log M\left(e^{\left(\psi\left(\frac{r}{2}\right)\right)^{\rho-\varepsilon_{1}}}, g\right) \geq \frac{\left(\psi\left(\frac{r}{2}\right)\right)^{\rho-\varepsilon_{1}}}{\log \frac{r}{2}} \log M(r, g) .
$$

This completes the proof of Lemma 2 .

The proof of Theorem 1. Suppose that $g(h)=P g+Q$ as stated in the theorem. Then we obtain that

$$
\begin{aligned}
\log M(r, g(h)) & =\log M(r, P g+Q) \\
& \leq \log \{M(r, P g)+M(r, Q)\} \\
& \leq \log M(r, P g)+\log M(r, Q)+\log 2 \\
& \leq \log M(r, P)+\log M(r, g)+\log M(r, Q)+O(1) .
\end{aligned}
$$

Let $t$ be the $\psi$-order of $P$ and put $\varepsilon_{1}=(1 / 2)(\rho$-t $)$.

Applying a result of G. Pólya $[\mathrm{P}]$ and Lemma 2, we have

$$
\log M(r, g(h)) \geq \log M\left(A_{1} e^{\left(\psi\left(\frac{r}{2}\right)\right)^{\rho-\varepsilon_{1}}}, g\right) \geq \frac{\left(\psi\left(\frac{r}{2}\right)\right)^{\rho-\varepsilon_{1}}}{\log r} \log M(r, g) .
$$

Since $\mathrm{P}$ has a $\psi$-order less than $\rho$, and $h$ has a lower $\psi$-order $\rho$, the last two inequalities above lead to a contradiction. This completes the proof of Theorem 1.

The proof of Theorem 2 is similar to Theorem 1, we omit it here. 


\section{On Further Study}

Remark 1. Obviously the result of Theorem 1 is not the best possible. Thus I would like to ask: "what is the optimal growth scale on $P$ such that it is still valid"; or ask "Can we find a growth-scale function, say, $\varphi:(1,+\infty) \longrightarrow(0,+\infty)$ in terms of growths of $g$ and $h$ such that (1)

$$
g(h)=P(z) g(z)+Q(z)
$$

where $P$ and $Q$ are entire functions with $\mathrm{M}(\mathrm{r}, \mathrm{Q})=\mathrm{o}(\mathrm{M}(\mathrm{r}, \mathrm{g}))$ as $r \longrightarrow$ $+\infty$, and

(2) $P$ has a $\varphi$-order $1 "$.

Remark 2. We do not deal with the case: $h$ is a transcendental entire function of logarithmic order one, it seems that Lemma 2 is not useful for functions of logarithmic order one. (Noticing that the composition function of two entire functions with finite logarithmic order is also of finite logarithmic order, in particular, any nonconstant polynomial is of logarithmic one.)

Remark 3. If we change the growth restriction condition on $Q$, then the decomposition of $g(h)$ in terms of $P g+Q$ may not be unique.

\section{References}

[B] H. Bohr, Über einen Sate von Edimund Lauda, Scripta Univ. Hierosolymitanarum, 1 (1923)

[C1] T. Y. Peter Chern, On meromorphic function with finite logarithmic order , Trans. Amer. Math. Soc. 358 (2006), no.2, 473-489.

[C2] T. Y. Peter Chern, On meromaphic functions, preprint, addressed in The second international conference of ISAAC, Fukuoka Institute of technology, Fukuoka, Kyushu, Japan (Aug. 16th - 20th, 1999).

[L] Yinian Lü, Dynamic systems of complex analysis, (in Chinese), Science Press 1995.

[N] R. Nevanlinna, Le Theoreme de Picard-Borel et la Theorie des Fonctions Meromorphes, Coll. Borel, 1929.

[P] G. Pólya, On an integral function of an integral function, J. London Math. Soc. $1(1926), 12-15$.

[T] E. C. Titchmarsh, The Theory of Functions, Oxford University Press, New York, 1939.

Peter Tien-Yu Chern

Department of Applied Mathematics, I-Shou University, 
Kaohsiung 84099, Taiwan, R.O.C.

E-mail: tychen@mail.isu.edu.tw

\section{In-Soo Kim}

Department of Mathematics, Institute of Pure and Applied Mathematics, Chonbuk National University,

Jeonju, Jeonbuk 561-756, Korea.

E-mail: insu@jbnu.ac.kr 